\title{
Solutions of Ginzburg-Landau equations and critical points of the renormalized energy
}

\author{
by
}

\author{
FANG HUA LIN*
}

Courant Institute of Mathematical Sciences

251 Mercer Street New York, NY 10012, USA

ABSTRACT. - Here we establish some connection between solutions of Ginzburg-Landau equations and critical points of the renormalized energy $W_{g}$ introduced in the recent works of Bethuel-Brezis and Helein. In particular, local nondegenerate minimums of $W_{g}$ have their associated solutions of GL-equations.

RÉsuMÉ. - On établit une relation entre les solutions de l'équation de Ginzburg-Landau et les points critiques de l'énergie renormalisée $W_{g}$ introduite dans les travaux récents de Bethuel-Brezis et Helein. En particulier, tout minimum local non dégénéré de $W_{g}$ est associé à une famille de solutions des équations de G-L.

\section{INTRODUCTION}

The aim of this article is to establish some connection between the solutions of Ginzburg-Landau Equations and critical points of the renormalized energy introduced in the recent work of Bethuel-Brezis-Helein [BBH]. We shall use the following Ginzburg-Landau heat flow as a basic

* Research is partially supported by an NSF-grant. 
link in proving such connections

$$
\begin{gathered}
u_{t}=\Delta u+\frac{1}{\epsilon^{2}}\left(1-|u|^{2}\right) u, \quad \text { in } \Omega \times \mathbb{R}_{+}, \\
u(x, t)=g(x), \quad \text { for } \quad x \in \partial \Omega, \quad t>0, \\
u(x, 0)=u_{0}(x), \text { for } x \in \Omega .
\end{gathered}
$$

Here $\Omega$ is a two-dimensional, smooth, bounded domain, $\epsilon$ is a positive parameter, $u: \Omega \rightarrow \mathbb{R}^{2}, g: \partial \Omega \rightarrow \mathbb{R}^{2}$ is smooth and $|g|=1$. We also assume that $u_{0}(x)=g(x)$ on $\partial \Omega$ and $\left|u_{0}(x)\right| \leq 1$. The motion of vortices of solutions $u$ of (1.1)-(1.3) will be our main concern. Based on formal asymptotics, various authors have arrived at the same conclusion that vortices move in the time scale of size $\log \frac{1}{\epsilon}$, see, e.g., $[\mathrm{N}],[\mathrm{E}]$, and $[\mathrm{PR}]$. Under some special assumptions, it was shown in $[R S]$ that it will take at least $0\left(\log \frac{1}{\epsilon}\right)$ time for a vortex to move a distance 1 . On the other hand, for the steady state solutions $u_{\epsilon}(x)$ of $(1.1)-(1.2)$ that is, the critical points of the functional

$$
E_{\epsilon}(u)=\frac{1}{2} \int_{\Omega}\left[|\nabla u|^{2}+\frac{1}{2 \epsilon^{2}}\left(1-|u|^{2}\right)^{2}\right] d x
$$

with $\left.u\right|_{\partial \Omega}=g$, a complete characterization of asymptotic behavior (as $\epsilon \rightarrow 0^{+}$) for vortices of $u_{\epsilon}$ is given in the recent book [BBH].

Let us recall one of the main results shown in $[\mathrm{BBH}]$ under the additional hypothesis that $\Omega$ is star-shaped. ( $C f$. also [St] when $\Omega$ is not star-shaped.)

THEOREM [BBH]. - Let $\Omega, g$ be as above and let $u_{\epsilon_{n}}$ be a sequence of steady state solutions of (1.1)-(1.2) (with $\epsilon=\epsilon_{n}$ ). Then there is a subsequence $\left\{u_{\epsilon_{n}}\right\}$ such that $u_{\epsilon_{n}}(x) \rightarrow u^{*}(x)$ in $C_{\text {loc }}^{1, \alpha}\left(\bar{\Omega} /\left\{a_{1}, a_{2}, \ldots, a_{k}\right\}\right), k \leq k(\Omega, g)$. Here $u^{*}(x)$ is given by

$$
\begin{gathered}
u^{*}(x)=\prod_{j=1}^{k}\left(\frac{z-a_{j}}{\left|z-a_{j}\right|}\right)^{d_{j}} e^{i h(x)}, z=x \in \Omega \subset \mathbb{C}, \\
\sum_{j=1}^{k} d_{j}=d=\quad \text { degree of } g
\end{gathered}
$$


and

$$
\Delta h=0 \text { in } \Omega,\left.\quad u^{*}\right|_{\partial \Omega}=g .
$$

For conveniences, we shall always assume $d \geq 0$.

If, in the addition, that $\left\{u_{\epsilon_{n}}\right\}$ are minimizers of (1.4), then in the above formula for $u^{*}, k=d, d_{j}=1$, for $j=1, \ldots, k$. Moreover, the point $a=\left(a_{1}, \ldots, a_{d}\right) \in \Omega^{d}$ is a global minimum point of the renormalized energy $W_{g}(b)$ defined on $\bar{\Omega}^{d}$ where for $b=\left(b_{1}, \ldots, b_{d}\right) \in \bar{\Omega}^{d}$,

$$
\begin{aligned}
W_{g}(b)= & -\pi \sum_{i \neq j} \log \left|b_{i}-b_{j}\right|+\frac{1}{2} \int_{\partial \Omega} \Phi\left(g \wedge g_{T}\right) \\
& -\pi \sum_{j=1}^{d} R\left(b_{j}\right),
\end{aligned}
$$

and $\Phi$ is the solution of

$$
\begin{aligned}
& \Delta \Phi=2 \pi \sum_{j=1}^{d} \delta_{b_{j}} \text { in } \Omega, \\
& \frac{\partial \Phi}{\partial \nu}=g \wedge g_{T} \text { on } \partial \Omega,
\end{aligned}
$$

$R$ is given by

$$
R(x)=\Phi(x)-\sum_{j=1}^{d} \log \left|x-b_{j}\right|
$$

From the above theorem, it is then natural to ask if there is a relation between the critical points of the function (1.4) and those of the renormalized energy $W_{g}(b), c f$. the open problems in $[\mathrm{BBH}]$. For this we have the following result.

THEOREM A. - Let $a=\left(a_{1}, \ldots, a_{d}\right) \in \Omega^{d}$ be a non-degenerate local minimum point for the renormalized energy $W_{g}$. Then there is a family $u_{\epsilon}, 0<\epsilon<\epsilon_{0}$, of steady state solutions of (1.1)-(1.2) such that

$$
u_{\epsilon}(x) \rightarrow u^{*}(x) \text { in } C_{\mathrm{loc}}^{1, \alpha}\left(\bar{\Omega} /\left\{a_{1}, \ldots, a_{d}\right\}\right)
$$

where

$$
u^{*}(x)=\prod_{j=1}^{d} \frac{x-a_{j}}{\left|x-a_{j}\right|} e^{i h(x)}, \Delta h=0 \quad \text { in } \quad \Omega
$$

and $u^{*}=g$ on $\partial \Omega$.

Vol. $12, n^{\circ}$ 5-1995. 
Here we say $a=\left(a_{1}, \ldots, a_{d}\right)$ is a nondegenerate local minimum of $W_{g}$ if

$$
\nabla W_{g}(a)=0 \text { and } \nabla^{2} W_{g}(a)
$$

is positive definite. In fact, we shall prove a somewhat more general result in which the hypothesis $\nabla^{2} W_{g}(a)$ being positive definite may be relaxed a bit.

The strategy of proving the above theorem is, for all sufficiently small $\epsilon$, to construct suitable initial data $u_{0}^{\epsilon}(x)$ and then solve (1.1)-(1.3) with $u(x, 0)=u_{0}^{\epsilon}(x)$. Let us denote $u_{\epsilon}(x)$ the asymptotic limit of $u(x, t)$ as $t \rightarrow \infty$, then $u_{\epsilon}(x)$ is the desired family for $0<\epsilon<\epsilon_{0}$. The key point in verifying the later statement is to control the locations of vortices of $u(x, t)$ for all $t \in(0, \infty)$. In general, we let $u_{\epsilon}(x, t)$ to denote the solution of (1.1)-(1.3). We consider the following two limits.

$$
\lim _{\epsilon \rightarrow 0} \lim _{t \rightarrow \infty} u_{\epsilon}(x, t)
$$

and

$$
\lim _{t \rightarrow \infty} \lim _{\epsilon \rightarrow 0} u_{\epsilon}(x, t) \text {. }
$$

For the initial data used in the proof of Theorem A, these two limits turn out to be the same. However, it is not the case for general initial data. In fact, these two limits may be characterized by the following.

THEOREM B.

(i) The $\lim _{t \rightarrow \infty} u_{\epsilon}(x, t)=u_{\epsilon}(x)$ exists, and $u_{\epsilon}(x)$ is a steady state solution of (1.1)-(1.2).

(ii) $\lim _{\epsilon \rightarrow 0} u_{\epsilon}(x)$ exists (up to subsequences). Moreover, these limits are those $u^{*}(x)$ of form given in (1.5). If we choose the initial data $u_{0}^{\epsilon}(x)$ suitably, then $k=d$, and $d_{j}=1$, for $j=1,2, \ldots, d$, in the formula (1.5). The singular points $a_{1}, \ldots, a_{d}$ are so that the point $a=\left(a_{1}, \ldots, a_{d}\right)$ is a critical point of $W_{g}$.

(iii) By choosing $u_{0}^{\epsilon}(x)$ properly, one has $\lim _{\epsilon \rightarrow 0} u_{\epsilon}(x, t)=u_{0}(x)$, and $u_{0}(x)$ is of form

$$
u_{0}(x)=\prod_{j=1}^{d} \frac{x-b_{j}}{\left|x-b_{j}\right|} e^{i h_{0}(x)}
$$

with $\Delta h_{0}(x)=0$ in $\Omega$. Here $b=\left(b_{1}, \ldots, b_{d}\right)$ can be an arbitrary point (depending on $u_{0}^{\varepsilon}$ ) of $\Omega^{d}$ such that $b_{i} \neq b_{j}$, for $i \neq j$.

The paper is written as follows. In the next section we shall start the proof of Theorem B and we shall establish various preliminary facts. Theorem A 
is proved in Section 3. Some of the arguments are employed to verify one statement needed in proving Theorem B, part (iii). This and some generalizations of Theorem A are discussed in the final section. Moreover, we shall also show the existence of mountain-pass type critical points of (1.4) whenever the renormalized energy $W_{g}$ has a critical point of the mountain-pass type.

\section{PROOF OF THEOREM B}

Under the forementioned hypothesis on $\Omega$, and $u_{0}(x)$ the global existence of a unique classical solution $u_{\epsilon}(x, t)$ for (1.1)-(1.3) follows from the standard parabolic theory, $c f$. [LSU]. Moreover, the solution $u_{\epsilon}(x, t)$ satisfies

$$
\frac{d}{d t} \int_{\Omega}\left[\left|\nabla u_{\epsilon}\right|^{2}+\frac{1}{2 \epsilon^{2}}\left(\left|u_{\epsilon}\right|^{2}-1\right)^{2}\right] d x=-2 \int_{\Omega}\left|\frac{d}{d t} u_{e}\right|^{2} d x .
$$

(2.1) implies, in particular, that

$$
\sup _{t>0} \int_{0}^{t} \int_{\Omega}\left|\frac{d}{d t} u_{\epsilon}(x, \tau)\right|^{2} d x d \tau+E_{\epsilon}\left(u_{\epsilon}(t)\right) \leq E_{\epsilon}\left(u_{0}\right) .
$$

Therefore, there is a sequence $\left\{t_{n}\right\}, t_{n} \rightarrow \infty$, such that $u_{\varepsilon}\left(x, t_{n}\right)$ converges, say in $C^{1, \alpha}$ norm, to a steady state solution $u_{\epsilon}(x)$ of (1.1)-(1.2). It follows from a deep theorem of $\mathrm{L}$. Simon [S] that $u_{\epsilon}(x, t)$ converges to $u_{\epsilon}(x)$ as $t \rightarrow \infty$ by the analyticity of $\left(|u|^{2}-1\right)^{2}$. This proves (i). The statement (ii) was proved in [BBH, Chapter 2]. In order to have $k=d, d_{j}=1, j=1,2, \ldots, d$ in (1.5), it suffices to construct the initial data $u_{0}^{\epsilon}(x)$ so that $E_{\epsilon}\left(u_{0}^{\epsilon}(x)\right) \leq \pi d \log \frac{1}{\epsilon}+C_{0}$, for some constant $C_{0}$. Indeed, in such a case, one also has $E_{\epsilon}\left(u_{\epsilon}(x)\right) \leq \pi d \log \frac{1}{\epsilon}+C_{0}$. The fact that $k=d$, and each $d_{j}$ is 1 for $j=1, \ldots, d$, follows from the arguments in [St] or [BBH]. To show (iii), we let $b_{1}, \ldots, b_{d}$ be arbitrary $d$-distinct points in $\Omega$, and let $u_{0}^{\epsilon}(x)$ be a function as $w(x)$ in the proof of [BBH, Lemma VIII.1]. That is, $u_{0}^{\epsilon}(x)=g(x)$ on $\partial \Omega$, and for some $\rho>0$,

$$
E_{\epsilon}\left(u_{0}^{\epsilon}\right) \leq d I(\epsilon, \rho)+W_{g}(b)+\pi d \log \frac{1}{\rho}+0(\rho)
$$

where

$0<\rho<\rho_{0}=\min \left\{\left|b_{i}-b_{j}\right|, \operatorname{dist}\left(b_{i}, \partial \Omega\right), i \neq j, \quad\right.$ for $\left.i, j=1,2, \ldots, d\right\}$.

For the definition of $I(\epsilon, \rho)$, see (3.4) below (cf. [BBH]). 
In particular, as $\epsilon \rightarrow 0$ and $\rho \rightarrow 0$, one has

$$
u_{0}^{\epsilon}(x) \rightarrow \prod_{j=1}^{d} \frac{x-b_{j}}{\left|x-b_{j}\right|} e^{i h_{0}(x)} \quad \text { in } \quad C_{\mathrm{loc}}^{1}\left(\bar{\Omega} \mid\left\{b_{1}, \ldots, b_{d}\right\}\right) .
$$

Here $\Delta h_{0}(x)=0$ in $\Omega$. Let $u_{\epsilon}(x, t)$ be the solution of (1.1)-(1.3) with the initial data $u_{0}^{\epsilon}(x)$ as above. Then we have the following estimates,

$$
\int_{0}^{\infty} \int_{\Omega}\left|\frac{d}{d t} u_{\epsilon}\right|^{2} d x d t \leq C ;(\text { a constant })
$$

$$
\int_{\Omega} \phi(x)\left[\left|\nabla u_{\epsilon}\right|(t)^{2}+\frac{1}{2 \epsilon^{2}}\left(\left|u_{\epsilon}\right|^{2}-1\right)(t)^{2}\right] d x \leq C(\phi) e^{t}, t>0 .
$$

Whose $\phi(x)$ in (2.5) should be chosen suitably, and here all the constants may depend on $g$ and $\Omega$ but they are independent of $\epsilon$.

The estimates (2.4) follow from the energy identity (2.1), the choice of $u_{0}^{\epsilon}(x)$ (so that (2.2) is true) and the lower energy bound discovered in [BBH]. In the estimates (2.5), $\phi$ can be any of the following functions, for $x_{0} \in \Omega /\left\{b_{1}, \ldots, b_{d}\right\}$, let $2 r=\rho\left(x_{0}\right)=\min _{1 \leq j \leq d}\left|x_{0}-b_{j}\right|$, and $\phi \in C_{0}^{1}\left(B_{r}\left(x_{0}\right)\right)$ with $\phi \equiv 1$ on $B_{r / 2}(x)$. In fact,

$$
\begin{aligned}
& \frac{d}{d t} \int_{\Omega} \phi^{2}\left[\left|\nabla u_{\epsilon}\right|^{2}+\frac{1}{2 \epsilon^{2}}\left(\left|u_{\epsilon}\right|^{2}-1\right)^{2}\right] d x \\
& \quad=-2 \int_{\Omega} \phi^{2}\left|\frac{d}{d t} u_{\epsilon}\right|^{2} d x+2 \int_{\Omega} \phi \nabla u_{\epsilon} \frac{d u_{\epsilon}}{d t} d x \\
& \quad \leq \int_{\Omega} \phi^{2}\left|\nabla u_{\epsilon}\right|^{2} d x+C(\phi) \int_{\Omega}\left|\frac{d}{d t} u_{\epsilon}\right|^{2} d x
\end{aligned}
$$

where $C(\phi)$ is simply $\|\nabla \phi\|_{L^{\infty}\left(\boldsymbol{B}_{r}\right)}$. (2.5) then follows from (2.6) by a simple integration and by using the estimate (2.4).

From (2.4) and (2.5), we obtain, for all $0<\epsilon<1$, that

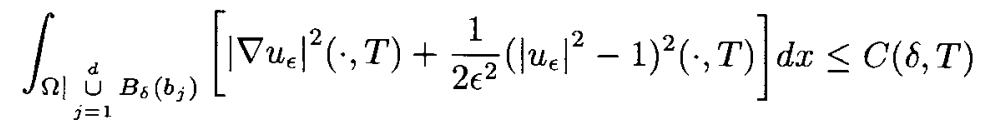

for any $\delta>0, T \in(0, \infty)$. 
We can now prove (1.10). First we observe that

$$
u_{\epsilon} \wedge \frac{d u_{\epsilon}}{d t}=\operatorname{div}\left(u_{\epsilon} \wedge \nabla u_{\epsilon}\right) \text { in } \Omega \times R_{+} .
$$

Since (2.4), (2.7), we see, for any sequence $\epsilon_{n} \downarrow 0$, there is a subsequence (still denoted by $\epsilon_{n}$ ) so that $u_{\epsilon_{n}}(x, t) \rightarrow u_{0}(x, t)$ weakly in $H_{\mathrm{loc}}^{\prime}\left(\bar{\Omega} /\left\{b_{1}, b_{2}, \ldots, b_{d}\right) \times R_{+}\right)$and strongly in $L_{\mathrm{loc}}^{2}\left(\bar{\Omega} \times R_{+}\right)$. The later is because that $\left|u_{\epsilon}(x, t)\right| \leq 1$. Moreover, $\left|u_{0}(x, t)\right|=1$ a.e. in $\Omega \times R_{+}$. We deduce from (2.8) and (1.1)-(1.3) that

$$
\left\{\begin{array}{l}
\frac{\partial u_{0}}{\partial t}=\Delta u_{0}+\left|\nabla u_{0}\right|^{2} u_{0} \text { in } \Omega /\left\{b_{1}, \ldots, b_{d}\right\} \times R_{+} \\
u_{0}(x, 0)=\prod_{j=1}^{d} \frac{x-b_{j}}{\left|x-b_{j}\right|} e^{i h_{0}(x)} \\
u_{0}=g \text { on } \partial \Omega \times R_{+}
\end{array}\right.
$$

Note that $u_{0}(x, t)$ is smooth in $\bar{\Omega} /\left\{b_{1}, \ldots, b_{d}\right\} \times \mathbb{R}_{+}$. Indeed, by (2.4), (2.7), one may deduce that, for any $t>0,0<\rho<$ $\rho_{0}, \operatorname{deg}\left(u_{0}(\cdot, t), \partial B_{\rho}\left(b_{j}\right)\right)=1$, for all $j=1, \ldots, d$. Hence, we may write

$$
u_{0}(x, t)=\prod_{j=1}^{d} \frac{x-b_{j}}{\left|x-b_{j}\right|} e^{i h(x, t)}
$$

$$
\text { with } h(x, t) \in H_{l o c}^{1}\left(\bar{\Omega} /\left\{b_{1} \ldots, b_{d}\right\} \times R_{+}\right)
$$

It is then clear that $h(x, t)$ satisfies the heat equation (1.10) away from $\left\{b_{1}, \ldots, b_{d}\right\} \times R_{+}$, and thus $u_{0}$ is smooth in $\Omega /\left\{b_{1}, \ldots b_{d}\right\} \times R_{+}$. Next, we observe that

$$
\begin{aligned}
\frac{d}{d t} \int_{B_{R}\left(b_{j}\right)}\left|x-b_{j}\right|^{2}\left[\frac{1}{2}\left|\nabla u_{\epsilon}\right|^{2}+\frac{1}{4 \epsilon^{2}}\left(\left|u_{\epsilon}\right|^{2}-1\right)^{2}\right] d x \\
=-\int_{B_{R}\left(b_{j}\right)}\left[\left|x-b_{j}\right|^{2}\left|\frac{d}{d t} u_{\epsilon}\right|^{2}+2\left(x-b_{j}\right) \cdot \nabla u_{\epsilon} \frac{d}{d t} u_{\epsilon}\right] d x \\
\quad+\int_{\partial B_{R}\left(b_{j}\right)}\left|x-b_{j}\right|^{2} \frac{\partial u_{\epsilon}}{\partial t} \cdot \frac{\partial u_{\epsilon}}{\partial \nu} \text { for } j=1,2, \ldots, d .
\end{aligned}
$$

Here $0<R<\rho_{1}=\min \left\{\rho_{0}, 1\right\}$. Since

$$
\begin{aligned}
& \int_{B_{R}\left(b_{i}\right)}\left(x-b_{j}\right) \nabla u_{\epsilon} \frac{d u_{\epsilon}}{d t} d x+\int_{\partial B_{R}\left(b_{j}\right)}\left(x-b_{j}\right) \nu e_{\epsilon}(u) \\
& -\int_{\partial B_{R}\left(b_{j}\right)}\left|x-b_{j}\right|\left(\frac{\partial u}{\partial \nu}\right)^{2}=\frac{1}{2 \epsilon^{2}} \int_{B_{R}\left(b_{j}\right)}\left(1-\left|u_{\epsilon}\right|^{2}\right)^{2} d x
\end{aligned}
$$

Vol. $12, n^{\circ}$ 5-1995. 
where $e_{\epsilon}(u)=\frac{1}{2}\left|\nabla u_{\epsilon}\right|^{2}+\frac{1}{4 \epsilon^{2}}\left(1-\left|u_{\epsilon}\right|^{2}\right)^{2}$ and $\nu$ is the exterior unit normal vector of $\partial B_{R}\left(b_{j}\right)$, we conclude from (2.11) that

$$
\begin{aligned}
\frac{d}{d t} \int_{B_{R}\left(b_{j}\right)} & \left|x-b_{j}\right|^{2} e_{\epsilon}(u) d x=-\int_{B_{R}\left(b_{i}\right)}\left|x-b_{j}\right|^{2}\left|\frac{d}{d t} u_{\epsilon}\right|^{2} d x \\
& -\int_{B_{R}\left(b_{j}\right)} \frac{1}{\epsilon^{2}}\left(1-\left|u_{\epsilon}\right|^{2}\right)^{2} d x \\
& +\int_{\partial B_{R}\left(b_{j}\right)}\left|x-b_{j}\right|^{2} \frac{\partial u_{\epsilon}}{\partial \nu} \frac{d}{d t} u_{\epsilon} \\
& +\int_{\partial B_{R}\left(b_{j}\right)}\left|x-b_{j}\right|\left(\frac{\left|\nabla_{T} u_{\epsilon}\right|^{2}}{2}+\frac{\left(\left|u_{\epsilon}\right|^{2}-1\right)^{2}}{4 \epsilon^{2}}\right) \\
& -\int_{\partial B_{R}} \frac{\left|x-b_{j}\right|}{2}\left|\frac{\partial u_{\epsilon}}{\partial \nu}\right|^{2} \\
\leq & \frac{-1}{\epsilon^{2}} \int_{B_{R}\left(b_{j}\right)}\left(1-\left|u_{\epsilon}\right|^{2}\right)^{2} d x \\
& +R \int_{\partial B_{R}\left(b_{j}\right)}\left[e_{\epsilon}(u)+\left|\frac{d}{d t} u_{\epsilon}\right|^{2}\right]
\end{aligned}
$$

Here we have used the fact $\left|x-b_{j}\right|^{2} \leq\left|x-b_{j}\right|$ as $R \leq 1$. By Fubini's Theorem, (2.7) and (2.13), we deduce, for each $j=1,2, \ldots, d$, that

$$
\begin{gathered}
\int_{B_{R}\left(b_{j}\right)}\left|x-b_{j}\right|^{2} e_{\epsilon}(u) d x+\int_{0}^{T} \int_{B_{R}\left(b_{j}\right)} \frac{1}{\epsilon^{2}}\left(1-\left|u_{\epsilon}\right|^{2}\right)^{2} d x \\
\leq R \int_{0}^{T} \int_{\partial B_{R}\left(b_{j}\right)}\left[e_{\epsilon}(u)+\left|\frac{d}{d t} u_{\epsilon}\right|^{2}\right] \leq C(R, T) .
\end{gathered}
$$

Here $R \in\left[\rho_{1} / 2, \rho_{1}\right]$ is chosen so that

$$
\begin{aligned}
& \int_{0}^{T} \int_{\partial B_{R}\left(b_{j}\right)}\left[e_{\epsilon}(u)+\left|\frac{d}{d t} u_{\epsilon}\right|^{2}\right] \\
& \quad \leq \frac{4}{R} \int_{0}^{T} \int_{B_{\rho_{1}} / B_{\rho_{1 / 2}}\left(b_{j}\right)}\left[e_{\epsilon}(u)+\left|\frac{d}{d t} u_{\epsilon}\right|^{2}\right] d x d t .
\end{aligned}
$$

Next we choose a small $\delta>0$ (its precise value will be determined later, see also discussion in the next section). Then there is $t \geq \epsilon_{0} \delta$ so that 
whenever $0 \leq t<t_{0}$, the set $\left\{x \in \Omega:\left|u_{\epsilon}(x, t)\right| \leq \frac{1}{2}\right\}$ is contained in $U_{j=1}^{d} B_{\delta}\left(b_{j}\right) \times\left[0, t_{0}\right]$. This follows from the small energy regularity theorems in [CS], [CL] and our energy estimates.

In the final section we should use arguments in the next section and estimates (2.4), (2.7) to show $h(x, t) \equiv h_{0}(x)$. The conclusion (1.10) then follows.

Remark. - It will be interesting to investigate also the case that $h_{0}(x)$ is not harmonic in $\Omega$.

\section{PROOF OF THEOREM A}

We first consider the case that $a=\left(a_{1}, \ldots, a_{d}\right)$ is a global nondegenerate minimum point for $W_{g}$ defined on $\bar{\Omega}^{d}$. Hence, in particular, one has

$$
\begin{gathered}
W_{g}(b)-W_{g}(a) \geq \lambda_{0}|b-a|^{2} \\
\text { whenever }|b-a| \leq \delta_{0}, \text { for some } \delta_{0}, \lambda_{0}>0 .
\end{gathered}
$$

By [BBH, Lemma VIII.1] and its proof, there is some $\rho_{0}>0$ (depending only on $a$ and $\Omega$ ) such that, for every $0<\rho \leq \rho_{0}$ and every $\epsilon>0$, one may find some $w_{\epsilon}(x)$ defined on $\Omega$ with $w_{\epsilon}=g$ on $\partial \Omega$ and

$$
E_{\epsilon}\left(w_{\epsilon}\right) \leq d I(\epsilon, \rho)+W_{g}(a)+\pi d \log \frac{1}{\rho}+0(\rho) .
$$

On the other hand, Lemma VIII 2 of [BBH] shows that

$$
\begin{gathered}
\min \left\{E_{\epsilon}(U):\left.U\right|_{\partial \Omega}=g\right\} \geq d I(\epsilon, \rho)+W_{g}(a) \\
+\pi d \log \frac{1}{\rho}+0\left(\rho^{2}\right), \text { whenever } \epsilon \leq \epsilon(\rho) .
\end{gathered}
$$

In inequalities (3.2) and (3.3), the quantity $I(\epsilon, \rho)$ is defined by

$$
\begin{gathered}
I(\epsilon, \rho)=\min \left\{\int_{B_{\rho}} \frac{1}{2}\left[|\nabla U|^{2}+\frac{1}{2 \epsilon^{2}}\left(|U|^{2}-1\right)^{2}\right] d x\right. \\
\left.u(x)=\frac{x}{|x|} \text { on }|x|=\rho\right\} .
\end{gathered}
$$


Next, we consider the heat-flow (1.1)-(1.3) with the initial data given by $w_{\epsilon}(x)$. Let us denote the solution of $(1.1)-(1.3)$ by $u_{\epsilon}(x, t)$. Then the following estimates hold:

$$
\begin{gathered}
-E_{\epsilon}\left(u_{\epsilon}(\cdot, t)\right)+E_{\epsilon}\left(w_{\epsilon}\right)=\int_{0}^{t} \int_{\Omega}\left|\frac{d}{d t} u_{\epsilon}(x, t)\right|^{2} d x d t \leq 0(\rho) \\
\left|\nabla u_{\epsilon}(x, t)\right|^{2}+\left|\frac{\partial}{\partial t} u_{\epsilon}(x, t)\right| \leq \frac{C}{\epsilon^{2}}, \text { for all }(x, t) \in \bar{\Omega} \times R^{+} .
\end{gathered}
$$

Here the number $\rho$ is suitably small but fixed, $0<\epsilon<\epsilon(\rho)$. The first estimate (I) follows easily from (3.2) and (3.3). The second estimate (II) can be easily obtained by consider the function $u_{\epsilon}\left(\epsilon x, \epsilon^{2} t\right)$ and the usual parabolic estimates. Of course, the initial data $w_{\epsilon}(x)$ should be chosen properly so that the estimate (II) will be valid for $0 \leq t \leq \epsilon$. This can be done and we leave it to the reader. In fact, from discussions in the previous section, we shall use (II) only for those $t \geq t_{0}$ (for some $t_{0}$ depending on $\delta_{0}$ ).

We let $\delta=\frac{1}{4 d} \min \left\{\delta_{0},\left|a_{j}-a_{i}\right|, i \neq j, i, j=1, \ldots, d\right\}$ and let $T_{\epsilon}>0$ be the first time that the set $\left\{x \in \Omega:\left|u_{\epsilon}\left(x, T_{\epsilon}\right)\right| \leq \frac{1}{2}\right\}$ interests the set $\bigcup_{j=1}^{d} \partial B_{\delta}\left(a_{j}\right)$. Suppose such time $T_{\epsilon}$ does not exist, then the time asymptotic $j=1$

limit $u_{\epsilon}(x)=\lim _{t \rightarrow \infty} u_{\epsilon}(x, t)$ will posses the property that the set

$$
\operatorname{deg}\left(u_{\epsilon}, \partial B_{\delta}\left(a_{j}\right)\right)=1, \quad j=1,2, \ldots, d .
$$

If (3.5) is true for all sufficiently small $\epsilon \in\left(0, \epsilon_{0}\right), \epsilon_{0}<\epsilon(\rho)$, then we are done. In fact, from the Theorem $[B B H]$ stated in the introduction we conclude that $u^{*}(x)=\lim _{\epsilon \rightarrow 0} u_{\epsilon}(x)$ exists (up to subsequence). Moreover, each $u^{*}(x)$ is of form

$$
u^{*}(x)=\prod_{j=1}^{d} \frac{x-\bar{a}_{j}}{\left|x-\bar{a}_{j}\right|} e^{i h(x)}
$$

for some

$$
\bar{a}=\left(\bar{a}_{1}, \ldots, \bar{a}_{d}\right), \quad \text { and } \quad\left|a_{j}-\bar{a}_{j}\right| \leq \delta .
$$

The last inequality follows from (3.5). By our assumption (3.1), we have $a=\bar{a}$ and therefore the conclusion of Theorem A. 
Suppose now such time $T_{\epsilon} \in(0, \infty)$ exists, for a sufficiently small $\epsilon$. We want to derive a contradiction from it. To avoid confusions that may arise from the sizes of various constants, we point out the order of these constants

$$
0<\epsilon \leq \epsilon_{0} \leq \epsilon(\rho)<<\rho<<\delta<\delta_{0} .
$$

We assume, without loss of the generality, that $\partial B_{\delta}\left(a_{1}\right) \cap\{x \in \Omega$ : $\left.\left|u_{\epsilon}\left(x, T_{\epsilon}\right)\right| \leq \frac{1}{2}\right\} \neq \phi$. Then there is an $x_{1}^{\epsilon} \in \Omega$ such that

$$
\left|u_{\epsilon}\left(x_{1}^{\epsilon}, T_{\epsilon}\right)\right|=\frac{1}{2},\left|x_{1}^{\epsilon}-a_{1}\right|=\delta .
$$

We choose $x_{j}^{\epsilon}, j=2, \ldots, d$, so that $\left|x_{j}^{\epsilon}-a_{j}\right| \leq \delta$ and $\left|u_{\epsilon}\left(x_{j}^{\epsilon}, T_{\epsilon}\right)\right| \leq \frac{1}{2}$. For each $j$, we may find $\alpha_{j} \in[\alpha, 2 \alpha], \alpha=\frac{1}{16 d+1}$, such that

$$
\epsilon^{\alpha_{j}} \int_{\partial B_{j}}\left[\left|\nabla V_{\epsilon}\right|^{2}+\frac{1}{2 \epsilon^{2}}\left(\left|V_{\epsilon}\right|^{2}-1\right)^{2}\right] \leq C(\alpha)
$$

where $B_{j}=\left\{x:\left|x-x_{j}^{\epsilon}\right| \leq \epsilon^{\alpha_{j}}\right\}, \epsilon(x)=u_{\epsilon}\left(x, T_{\epsilon}\right)$, and $C(\alpha)$ is a constant depending only on $\alpha, g$ and $\Omega$. The estimates (3.9) is an easy consequence of the Fubini's Theorem (3.2), and the energy estimate (I), see also [St]. Next, we let $\tilde{U}_{\epsilon}(x)$ be such that it minimizes

$$
\int_{\Omega_{\epsilon}}\left[|\nabla u|^{2}+\frac{1}{2 \epsilon^{2}}\left(|u|^{2}-1\right)^{2}\right] d x
$$

with $\tilde{U}_{\epsilon}=V_{\epsilon} \quad$ on $\quad \partial \Omega_{\epsilon}$. Where $\Omega_{\epsilon}=\Omega / \bigcup_{j=1}^{d} B_{j}$. Now we claim the following two statements are true:

$$
\begin{gathered}
\left|\tilde{U}_{\epsilon}(x)\right| \geq \frac{1}{2} \quad \text { on } \Omega_{\epsilon} ; \\
\operatorname{deg}\left(V_{\epsilon}, \partial B_{j}\right)=1, \quad \text { for } j=1, \ldots, d .
\end{gathered}
$$

These two statements should be proved later. Suppose, for the moment, that they are both true. Then, as $\epsilon \rightarrow 0$, we may assume, by taking subsequences if necessary, that

$$
x_{j}^{\epsilon} \rightarrow \bar{a}_{j}, \quad \text { for } j=1, \ldots, d, \text { and }\left|\bar{a}_{1}-a_{1}\right|=\delta .
$$

Vol. $12, n^{\circ}$ 5-1995. 
Moreover, the corresponding subsequence of $\tilde{U}_{\epsilon}(x)$ converges to $\bar{U}(x)=$ $\prod_{j=1}^{d} \frac{x-\bar{a}_{j}}{\left|x-\bar{a}_{j}\right|} e^{i H(x)}$ in $C_{\mathrm{loc}}^{1, \alpha}\left(\bar{\Omega} /\left\{\bar{a}_{1}, \ldots, \bar{a}_{d}\right)\right.$ with $\Delta H(x)=0$. The later statement follows easily from the assertions (3.10), (3.11).

In fact, for each $x \in \Omega_{\epsilon}$, we may find a disc $D$ of radius $\epsilon^{\beta}$, for some $\beta \in[3 \alpha, 4 \alpha]$, such that

$$
x \in D \cap \Omega_{\epsilon}, \epsilon^{\beta} \int_{\partial D \cap \Omega_{\epsilon}}\left[\left|\nabla \tilde{u}_{\epsilon}\right|^{2}+\frac{1}{2 \epsilon^{2}}\left(\left|\tilde{u}_{\epsilon}\right|^{2}-1\right)\right] \leq c(\alpha)
$$

(see (3.9)). Moreover, $\frac{1}{\epsilon^{\beta}}\left(D \cap \Omega_{\epsilon}\right)$ is a Lipschitz domain with Lipschitz character independent of $\epsilon$. On $\frac{1}{\epsilon^{\beta}}\left(D \cap \Omega_{\epsilon}\right)=D_{\epsilon}$, the function $\tilde{u}_{\epsilon}\left(\epsilon^{\beta} x\right)$ minimizes the functional of form

$$
\int_{D_{\epsilon}}\left[|\nabla u|^{2}+\frac{1}{2 \epsilon^{2(1-\beta)}}\left(|u|^{2}-1\right)\right] d x
$$

and with boundary data $g_{\epsilon}$ on $\partial D_{\epsilon}$ satisfying

$$
\int_{\partial D_{\epsilon}}\left[\left|\nabla_{T} g_{\epsilon}\right|^{2}+\frac{1}{2 \epsilon^{2(1-\beta)}}\left(\left|g_{\epsilon}\right|^{2}-1\right)^{2}\right] \leq C(\alpha) \text {. }
$$

Since $\left|\tilde{u}_{\epsilon}\right| \geq \frac{1}{2}$ on $D \cap \Omega_{\epsilon}$, one has also that

$$
\operatorname{deg}\left(g_{\epsilon}, \partial D_{\epsilon}\right)=0 .
$$

We write $\tilde{u}_{\epsilon}\left(\epsilon^{\beta} x\right)=\rho_{\epsilon} e^{i \psi_{\epsilon}}$ on $D_{\epsilon}$. Then it is clear that $\psi_{\epsilon}$ minimizes $\int_{D_{\epsilon}} \rho_{\epsilon}^{2}\left|\nabla \psi_{\epsilon}\right|^{2} d x$ and with $\psi_{\epsilon} \in H^{\prime}\left(\partial D_{\epsilon}\right)$. Thus, $\nabla \psi_{\epsilon} \in L^{p}\left(D_{\epsilon}\right)$ for some $p>2$.

Then the function $f_{\epsilon}(x)=1-\rho_{\epsilon}(x)$ satisfies

$$
-\Delta f_{\epsilon}(x)+\frac{c_{\epsilon}(x)}{\epsilon^{2(1-\beta)}} f_{\epsilon}(x)+p_{\epsilon}(x)=0
$$

in $D_{\epsilon}$ with $0 \leq f_{\epsilon} \leq \frac{1}{2}, \frac{1}{4} \leq c_{\epsilon}(x) \leq 4$, and $p_{\epsilon}(x) \in L^{q}\left(D_{\epsilon}\right)$, for some $q>1$. Moreover, on $\partial D_{\epsilon}, f_{\epsilon} \leq c \epsilon^{\frac{1}{4}}$, by (3.12). Therefore, elliptic estimates yield $f_{\epsilon} \leq c \epsilon^{\frac{1}{4}}+c \epsilon^{q-1}$.

We now write $\tilde{u}_{\epsilon}(x)$ on $\Omega_{\epsilon}$ as

$$
\tilde{u}_{\epsilon}(x)=\prod_{j=1}^{d} \frac{x-x_{j}^{\epsilon}}{\left|x-x_{j}^{\epsilon}\right|} e^{i h_{\epsilon}(x)} \cdot \tilde{\rho}_{\epsilon}(x)
$$


with $1-c \epsilon^{\frac{1}{4}}-c \epsilon^{q-1} \leq \tilde{\rho}_{\epsilon}(x) \leq 1$. Let $\Theta_{\epsilon}(x)$ be such that $e^{i \Theta_{\epsilon}(x)}=$ $\prod_{j=1}^{d} \frac{x-x_{j}^{\epsilon}}{\left|x-x_{j}^{\epsilon}\right|}$, then $\Theta_{\epsilon}$ is a multivalued harmonic function in $\Omega_{\epsilon}$, and $h_{\epsilon}(x)$ minimizes the integral

$$
\int_{\Omega_{\epsilon}}\left[\tilde{\rho}_{\epsilon}^{2}\left|\nabla h_{\epsilon}\right|^{2}+2 \tilde{\rho}_{\epsilon}^{2} \nabla h_{\epsilon} \cdot \nabla \Theta_{\epsilon}\right] d x
$$

We want to show $\int_{\Omega_{\epsilon}} \tilde{\rho}_{\epsilon}^{2}\left|\nabla h_{\epsilon}\right|^{2} d x \leq c<\infty$. It then follows that $\nabla h_{\epsilon} \in L_{\text {loc }}^{p}(\Omega)$ for some $p>2$. The convergence of $\tilde{u}_{\epsilon}$ to $\bar{u}(x)$ follows easily as in [BBH, Chapter VI].

To show $\int_{\Omega_{\epsilon}}\left|\nabla h_{\epsilon}\right|^{2} d x \leq c$, we first choose a function $\tilde{h}_{\epsilon}(x)$ on $\Omega_{\epsilon}$ such that $\tilde{h}_{\epsilon}(x) \equiv 0$ on $\Omega \mid\left(\bigcup_{j=1}^{d} 2 B_{j}\right)$ where $\Omega_{\epsilon}=\Omega \mid \bigcup_{j=1}^{d} B_{j}$, and $2 B_{j}$ is the double of $B_{j}$ (with the same center), $j=1, \ldots, d$. Moreover, $\int_{\Omega_{\varepsilon}}\left|\nabla \tilde{h}_{\epsilon}\right|^{2} \leq c(\alpha, g),\left|\tilde{h}_{\epsilon}\right| \leq c(\alpha, g)$. The later can be achieved due to the conformal invariant of the Dirichlet integral and the fact that

$$
\int_{\partial \Omega_{\epsilon}}\left|\nabla u_{\epsilon}\right|^{2}+\frac{1}{2 \epsilon^{2}}\left(1-\left|u_{\epsilon}\right|^{2}\right)^{2} \leq c(\alpha, g)
$$

Therefore, by minimality, one has

$$
\begin{aligned}
& \int_{\Omega_{\epsilon}}\left[\tilde{\rho}_{\epsilon}^{2}\left|\nabla h_{\epsilon}\right|^{2}+2 \tilde{\rho}_{\epsilon}^{2} \nabla h_{\epsilon} \cdot \nabla \Theta_{\epsilon}\right] d x \\
& \quad \leq \int_{\Omega_{\epsilon}}\left[\tilde{\rho}_{\epsilon}^{2}\left|\nabla \tilde{h}_{\epsilon}\right|^{2}+2 \tilde{\rho}_{\epsilon}^{2} \nabla \tilde{h}_{\epsilon} \cdot \nabla \Theta_{\epsilon}\right] d x
\end{aligned}
$$

Next we wish to show $\int_{\Omega_{\epsilon}} \nabla h_{\epsilon} \cdot \nabla \Theta_{\epsilon} d x$ and $\int_{\Omega_{\epsilon}} \nabla \tilde{h}_{\epsilon} \nabla \Theta_{\epsilon} d x$ are uniformly bounded. If we show this, then we deduce from the above inequality $*$ and the estimate $\tilde{\rho}_{\epsilon} \geq 1-c \epsilon^{\frac{1}{4}}-c \epsilon^{q-1}$ that

$$
\int_{\Omega_{\epsilon}} \tilde{\rho}_{\epsilon}^{2}\left|\nabla h_{\epsilon}\right|^{2} d x \leq c(\alpha, g)+\int_{\Omega_{\epsilon}} \tilde{\rho}_{\epsilon}^{2}\left|\nabla \tilde{h}_{\epsilon}\right|^{2} d x .
$$

where we noticed that $|\nabla \Theta|(x)\left(1-\tilde{\rho}_{(x)}\right) \leq c\left(\epsilon^{\frac{1}{8}}+\epsilon^{q-1-\alpha}\right)$ and we may assume $\alpha<q-1$. 
Finally, since $\Theta_{\epsilon}$ is harmonic in $\Omega_{\epsilon}$, one has

$$
\int_{\Omega_{\epsilon}} \nabla h_{\epsilon} \cdot \nabla \Theta_{\epsilon} d x=\sum_{j=1}^{d} \int_{\partial B_{j}} h_{\epsilon} \frac{\partial \Theta \epsilon}{\partial \nu}+\int_{\partial \Omega} h_{\epsilon} \frac{\partial \Theta \epsilon}{\partial \nu} .
$$

As we may always assume $\left.h_{\epsilon}\right|_{\partial \Omega}$ is bounded (depending only on $g$ ) the last term in the above equation is clearly bounded.

Next, for each $j$, we write $\Theta_{\epsilon}(x)=\theta_{j}(x)+\psi_{j}^{\epsilon}(x), \theta_{j}(x)$ is the argument of $\left(x-x_{\epsilon}^{j}\right)$. Then, since $\frac{\partial \theta_{j}}{\partial \nu}=0$ on $\partial B_{j}$, and $\psi_{j}^{\epsilon}(x)$ is harmonic inside $B_{j}$, we have

$$
\int_{\partial B_{j}} h_{\epsilon} \frac{\partial \Theta_{\epsilon}}{\partial \nu}=\int\left(h_{\epsilon}-\bar{h}_{\epsilon}\right) \frac{\partial \psi^{\epsilon}}{\partial \nu}
$$

where $\bar{h}_{\epsilon}$ is the average of $h_{\epsilon}$ on $\partial B_{j}$.

Now, via the fact that $o s c h_{\epsilon}$ on $\partial B_{j}$ is uniformal bounded, we see each $\int_{\partial B_{j}} h_{\epsilon} \frac{\partial \Theta_{\epsilon}}{\partial \nu} \rightarrow 0$ as $\epsilon \rightarrow 0$. This completes the proof of our statement that $\int_{\Omega_{\epsilon}}\left|\nabla h_{\epsilon}\right|^{2} d x \leq c(\alpha, g)$.

It then also follows from the definition of $W_{g}$ in $[\mathrm{BBH}]$, that $\frac{1}{2} \int_{\Omega_{\rho}(\bar{a})}\left[\left|\nabla \tilde{U}_{\epsilon}\right|^{2}+\frac{1}{2 \epsilon^{2}}\left(\left|\tilde{U}_{\epsilon}\right|^{2}-1\right)^{2}\right] d x=\pi d \log \frac{1}{\rho}+W_{g}(\bar{a})+0(\rho)+o(1)$ Here $o(1) \rightarrow 0$ as $\epsilon \rightarrow 0$, and $\rho<\delta$ is a fixed constant. Finally, to complete our arguments, we look at the comparison map $\bar{V}_{\epsilon}(x)$,

$$
\bar{V}_{\epsilon}(x)= \begin{cases}V_{\epsilon}(x), & x \in \bigcup_{j=1}^{d} B_{j}, \\ \tilde{U}_{\epsilon}(x) & x \in \Omega_{\epsilon} .\end{cases}
$$

Then, on the one hand, we have

$$
\begin{aligned}
& E_{\epsilon}\left(\bar{V}_{\epsilon}\right) \leq E_{\epsilon}\left(V_{\epsilon}\right) \leq E_{\epsilon}\left(w_{\epsilon}\right) \leq \pi d \log \frac{1}{\rho} \\
& \quad+\min W_{g}+d I(\epsilon, \rho)+0(\rho)
\end{aligned}
$$

and, on the other hand

$$
\begin{aligned}
& E_{\epsilon}\left(\bar{V}_{\epsilon}\right)=\pi d \log \frac{1}{\rho}+W_{g}(\bar{a})+0(\rho)+o(1) \\
& \quad+\sum_{j=1}^{d} \frac{1}{2} \int_{B_{\rho}\left(\bar{a}_{j}\right)}\left[\left|\nabla \bar{V}_{\epsilon}\right|^{2}+\frac{1}{2 \epsilon^{2}}\left(\left|\bar{V}_{\epsilon}\right|^{2}-1\right)^{2}\right] d x .
\end{aligned}
$$


By the convergence of $\tilde{U}_{\epsilon}$ to $\bar{U}(x)$ and by the definition of $I(\epsilon, \rho)$, we see the last term on the right hand side of (3.16) is larger than $d I(\epsilon, \rho)+0(\rho)$, That is

$$
E_{\epsilon}\left(\bar{V}_{\epsilon}\right) \geq \pi d \log \frac{1}{\rho}+W_{g}(\bar{a})+0(\rho)+o(1)+d I(\epsilon, \rho) .
$$

We observe that $|\bar{a}-a| \cong \delta<\delta_{0}$, thus (3.1) yields $W_{g}(\bar{a}) \geq W_{g}(a)+C \delta^{2}$ for some $C>0$.

(3.16) and (3.17) lead to a contradiction if we choose $\rho$ is much small than $\delta^{2}$ to start with. This proves Theorem $\mathrm{A}$, in the case $a$ is a global nondegenerate minimum point for $W_{g}$, modulo the statements (3.10) and (3.11).

Remark. - Under the assumption (3.1), we first choose $\delta=$ $\frac{1}{4 d} \min \left\{\delta_{0},\left|a_{i}-a_{j}\right|, i \neq j\right\}$. Then we fix $\rho<<\delta^{2}$ so that various $0(\rho)$ terms can be bounded by $\frac{\lambda_{0}}{4 d}|a-\bar{a}|^{2}$. Finally we choose $\epsilon_{0}<\epsilon(\rho)$ to make all of the arguments go through.

Remark. - The assumption that $a$ is a global minimum point of $W_{g}$ is used only to conclude (3.10) and (3.11). When $a$ is a local nondegenerate minimum point, we should introduce another argument to obtain the similar statements as (3.10) and (3.11).

Proof of (3.10) and (3.11).

We start with the following lemmas.

Lemma 1. - Let $u$ be a minimizer of the functional

$$
E_{\epsilon}(u)=\frac{1}{2} \int_{B}\left[|\nabla u|^{2}+\frac{1}{2 \epsilon^{2}}\left(|u|^{2}-1\right)^{2}\right] d x
$$

in the unit disc $B$ with $u=g$ on $\partial B$. Suppose that

$$
\int_{\partial B}\left[\left|\nabla_{T} g\right|^{2}+\frac{1}{2 \epsilon^{2}}\left(|g|^{2}-1\right)^{2}\right] \leq C_{1}
$$

for a constant $C_{1}$. Then, for all sufficiently small $\epsilon>0$ (depending only on $C_{1}$ ) we have

$$
E_{\epsilon}(u) \leq C_{2} \text { (depending only on } C_{1} \text { ) }
$$

whenever $\operatorname{deg}(g, \partial B)=0$, and

$$
E_{\epsilon}(u) \geq \pi|d| \log \frac{1}{\epsilon}+C_{3}
$$

if $\operatorname{deg}(g, \partial B)=d \neq 0$.

Vol. 12, $\mathrm{n}^{\circ}$ 5-1995. 
Proof. - From (3.18) one easily deduces that $g \in C^{1 / 2}(\partial B)$ and $|g| \geq 1-C \epsilon^{1 / 4}$ on $\partial B$, for a constant $C$ depending on $C_{1}$. In particular, the $\operatorname{deg}(g, \partial B)$ is well-defined. In the case $\operatorname{deg}(g, \partial B)=0$, we may write $g(\theta)=\rho(\theta) e^{i \psi(\theta)}$ on $\partial B$. Let $\psi^{*}$ be the harmonic extension of $\psi$ into $B$, and $\rho^{*}(r, \theta) \equiv 1$ on $B_{1-\epsilon}, \rho^{*}(r, \theta)$ is linear in $r \in[1-\epsilon, 1]$ for each $\theta$. Then let $U^{*}=\rho^{*} e^{i \psi^{*}}$, we have

$$
E_{\epsilon}\left(U^{*}\right) \leq \frac{1}{2} \int_{B}\left|\nabla \psi^{*}\right|^{2} d x+C \epsilon=C_{2} .
$$

This is (3.19). The estimate (3.20) is much hard to prove but it can be found in $[\mathrm{BBH}]$.

Lemma 2. - With the same hypothesis as Lemma 1, and suppose $\operatorname{deg}(g, \partial B)=0$ Then $|u(x)| \geq \frac{3}{4}$ in $B$ whenever $0<\epsilon \leq \epsilon_{1}$, for some $\epsilon_{1}$.

Proof. - Suppose not, we would have a sequence $\epsilon_{n} \downarrow 0$, and a sequence of minimizers $u_{\epsilon_{n}}=u_{n}$ with boundary data $g_{n}$ satisfying (3.18) and $\operatorname{deg}\left(g_{n}, \partial B\right)=0$. Moreover, inf $\left|u_{n}\right| \leq \frac{3}{4}$.

Since $\left|g_{n}\right| \rightarrow 1,\left\|g_{n}\right\|_{C^{1 / 2}(\partial B)} \leq C$, we see $|u|(x) \geq \frac{4}{5}>\frac{3}{4}$ whenever $1-|x| \leq C_{0} \epsilon_{n}$, for some $C_{0}$. Indeed, the function $V_{n}(x)=u_{n}\left(\epsilon_{n} x\right)$ satisfies (a) $\left|V_{n}(x)-V_{n}(y)\right| \leq C|x-y|^{1 / 2}$ whenever $|x-y| \leq 1$ and $x, y \in \frac{1}{\epsilon_{n}} B$; and $(b)\left|\nabla V_{n}(x)\right| \leq \frac{C}{R}$ for $R \in(0,1)$ and $|x| \leq \frac{1}{\epsilon_{n}}-R$. Both (a) and (b) follow from the standard elliptic estimates.

Hence, if $\left|u_{n}\left(x_{n}\right)\right| \leq \frac{3}{4}$, then there is a ball $\left\{x:\left|x-x_{n}\right| \leq \eta \epsilon_{n}\right\} \subset B$, for some $\eta>0$ with $\left|u_{n}(x)\right| \leq \frac{4}{5}$ for all $x,\left|x-x_{n}\right| \leq \eta \epsilon_{n}$. Therefore

$$
\int_{B} \frac{1}{\epsilon_{n}^{2}}\left(\left|u_{n}\right|^{2}-1\right)^{2} d x \geq C(\eta)>0 .
$$

By Lemma $1, E_{\epsilon_{n}}\left(u_{n}\right) \leq E_{\epsilon_{n}}\left(U_{n}^{*}\right) \leq C_{2}$. Since $g_{n} \rightarrow g=e^{i \psi}$ weakly in $H^{\prime}(\partial B), \int_{B}\left|\nabla \psi_{n}^{*}\right|^{2} d x$ converges to $\int_{B}\left|\nabla \psi^{*}\right|^{2} d x$, where $\psi^{*}$ is the harmonic extension of $\psi$. Thus $\overline{\lim } E_{\epsilon_{n}}\left(U_{n}^{*}\right) \leq \frac{1}{2} \int_{B}\left|\nabla \psi^{*}\right|^{2} d x$. On the other hand, $u_{n} \rightarrow u$ weakly in $H^{\prime}(B)$ with $u=g$ on $\partial B$ and $|u|=1$ a.e. in $\mathrm{B}$. We have

$$
\underline{\lim } E_{\epsilon_{n}}\left(u_{n}\right) \geq C(\eta)+\lim _{n} \frac{1}{2} \int_{B}\left|\nabla u_{n}\right|^{2} \geq C(\eta)+\frac{1}{2} \int_{B}\left|\nabla \psi^{*}\right|^{2} .
$$


We therefore obtain a contradiction.

Remark. - Both lemma 1 and lemma 2 remain true when we replace $B$ by a bounded Lipschitz domain.

To prove (3.10) and (3.11), we first show $\left|\operatorname{deg}\left(V_{\epsilon}, \partial B_{j}\right)\right|=1$, for $j=1,2, \ldots d$. Indeed, $\left|\operatorname{deg}\left(V_{\epsilon}, \partial B_{j}\right)\right| \geq 1$, for $j=1,2, \ldots, d$, for otherwise we may replace $V_{\epsilon}$ in one of the ball $B_{j}$ by a new map $\tilde{V}_{\epsilon}$ that minimizes the $E_{\epsilon}(\cdot)$ on $B_{j}$ and $\tilde{V}_{\epsilon}=V_{\epsilon}$ on $\partial B_{j}$. Since $\left|V_{\epsilon}\left(x_{j}^{\epsilon}\right)\right| \leq \frac{1}{2}$ and since $\left|\nabla V_{\epsilon}\right| \leq \frac{C}{\epsilon}$, we conclude as in the Lemma 2 that

$$
\begin{aligned}
& \int_{B_{j}}\left[\left|\nabla V_{\epsilon}\right|^{2}+\frac{1}{2 \epsilon^{2}}\left(\left|V_{\epsilon}\right|^{2}-1\right)^{2}\right] d x \\
& \geq \int_{B_{j}}\left[\left|\nabla \tilde{V}_{\epsilon}\right|^{2}+\frac{1}{2 \epsilon^{2}}\left(\left|\tilde{V}_{\epsilon}\right|^{2}-1\right)^{2}\right]+C_{0}
\end{aligned}
$$

for some $C_{0}>0$ (see (3.21)). This will contradict the fact that (3.2), (3.3) and the estimate (I).

By (3.20), we have

$$
\begin{aligned}
& \frac{1}{2} \sum_{j=1}^{d} \int_{B_{j}}\left[\left|\nabla V_{\epsilon}\right|^{2}+\frac{1}{2 \epsilon^{2}}\left(\left|V_{\epsilon}\right|^{2}-1\right)^{2}\right] d x \\
& \quad \geq \pi \sum_{j=1}^{d}\left(1-\alpha_{j}\right)\left|\operatorname{deg}\left(V_{\epsilon}, \partial B_{j}\right)\right| \log \frac{1}{\epsilon}+0(1)
\end{aligned}
$$

(by our choice of $\left.\alpha_{j}\right) \geq \pi\left(d-\frac{1}{8}\right) \log \frac{1}{\epsilon}+0(1)$. This combined with (3.2) implies each $\left|\operatorname{deg}\left(V_{\epsilon}, \partial B_{j}\right)\right|=1$. Next, we want to show (3.10). If for some $x_{\epsilon} \in \Omega_{\epsilon},\left|\tilde{U}_{\epsilon}\left(x_{\epsilon}\right)\right| \leq \frac{1}{2}$, then there are two possibilities: either $\operatorname{dist}\left(x_{\epsilon}, \partial \Omega_{\epsilon}\right) \geq \epsilon^{3 \alpha}$ or $\operatorname{dist}\left(x_{\epsilon}, \partial \Omega_{\epsilon}\right)<\epsilon^{3 \alpha}$. In the first case, we find a ball $D$ around $x_{\varepsilon}$ of radius $\epsilon^{\beta}, \beta \in(3 \alpha, 4 \alpha)$ such that the similar estimate as (3.9) is true. Then if $\operatorname{deg}\left(\tilde{U}_{\epsilon}, \partial D\right)=0$, we obtain a contradiction from Lemma 2 and the fact that $\tilde{U}_{\epsilon}$ is already a minimizer on $D$. If $\operatorname{deg}\left(\tilde{U}_{\epsilon}, \partial D\right) \neq 0$, then the energy of $\tilde{U}_{\epsilon}$ on $\mathrm{D}$ will be larger than $\pi(1-4 \alpha) \log \frac{1}{\epsilon}+0(1)$. This contradicts the fact that the total energy of $\tilde{U}_{\epsilon}$ on $\Omega_{\epsilon}$ is not larger than $\frac{\pi}{8} \log \frac{1}{\epsilon}+0(1)$. In the second case, we may find a point $y_{\epsilon} \in \partial \Omega_{\epsilon},\left|x_{\epsilon}-y_{\epsilon}\right|<\epsilon^{3 \alpha}$. We again find a ball $D$ around $y_{\epsilon}$ Vol. $12, n^{\circ}$ 5.1995. 
of radius $\epsilon^{\beta}$, for some $\beta \in\left(\frac{5}{2} \alpha, 3 \alpha\right)$ so that

$$
\epsilon^{\beta} \int_{\partial D \cap \Omega \epsilon}\left[\left|\nabla \tilde{U}_{\epsilon}\right|^{2}+\frac{1}{2 \epsilon^{2}}\left(\left|\tilde{U}_{\epsilon}\right|^{2}-1\right)^{2}\right] d x \leq C(\alpha) .
$$

Since the domain $\frac{1}{\epsilon^{\beta}}\left(D \cap \Omega_{\epsilon}\right)$ is clearly a bounded Lipschitz domain as $\beta \geq \frac{5 \alpha}{2}$, we apply Lemma 1 and Lemma 2 again to obtain a contradiction as in the first case. We have therefore completed the proof of Theorem $A$ under the assumption that $a$ is also a global minimum point.

We turn now to the case that $a$ is only a nondegenerate minimum point. We need to modify the part showing the statements (3.20) and (3.11). The key point is that we may not have

$$
\operatorname{deg}\left(V_{\epsilon}, \partial B_{j}\right) \neq 0, \text { for all } j=1,2, \ldots d
$$

Indeed, if we have (3.22), then above arguments apply to conclude that $\left|\tilde{U}_{\epsilon}(x)\right| \geq \frac{1}{2}$ on $\Omega_{\epsilon}$ and by (3.2), we must have $\operatorname{deg}\left(V_{\epsilon}, \partial B_{j}\right)=1$, for $j=1,2, \ldots, d$. Here one notices also that, since

$$
\left|\tilde{U}_{\epsilon}(x)\right| \geq \frac{1}{2}, d=\sum_{j=1}^{d} \operatorname{deg}\left(V_{\epsilon}, \partial B_{j}\right) \leq \sum_{j=1}^{d}\left|\operatorname{deg}\left(V_{\epsilon}, \partial B_{j}\right)\right| \leq d .
$$

Then as $\operatorname{deg}\left(V_{\epsilon}, \partial B_{j}\right)=1$, and $\left|\tilde{U}_{\epsilon}\right|(x) \geq \frac{1}{2}, x \in \Omega_{\epsilon}$, we may apply the same arguments as before to obtain

$$
W_{g}(\bar{a}) \leq W_{g}(a)+0(\rho)+o(1)
$$

where $\epsilon \leq \epsilon_{0}$. Here $\left|a_{1}-\bar{a}_{1}\right|=\delta,\left|a_{j}-\bar{a}_{j}\right| \leq \delta$. (cf. (3.15), (3.16) and (3.17)). (3.23) will contradict to (3.1) whenever $\rho$ is much smaller than $\delta^{2}$ and $\epsilon$ is sufficiently small.

Therefore, (3.22) can not be true for all $j$. Note that by our choice of $T_{\epsilon}$, we have $\operatorname{deg}\left(V_{\epsilon}, B_{\delta}\left(a_{j}\right)\right)=1$, for $j=1,2, \ldots, d$.

From now on, the first ball $B_{1}$ with center lies at distance $\delta$ form $a_{1}$ will be no longer used. We may assume, without loss of the generality, that $\operatorname{deg}\left(V_{\epsilon}, \partial B_{1}\right)=0$. We then change $V_{\epsilon}$ inside $B_{1}$ by a minimizer of $E_{\epsilon}(\cdot)$ with the same boundary value as $V_{\epsilon}$. Since the estimate (II), Lemma 2, (3.21) we see the energy of the new map (which is $V_{\epsilon}$ outside $B_{1}$ and is the minimizer inside $B_{1}$ ), which we call $V_{\epsilon}^{\prime}$, will satisfy $E_{\epsilon}\left(V_{\epsilon}^{\prime}\right) \leq E_{\epsilon}\left(V_{\epsilon}\right)-C_{0}$, 
for some positive constant $C_{0}$. We then pick up another point, say $x_{1}$, inside $B_{\delta}\left(a_{1}\right)$ such that $\left|V_{\epsilon}^{\prime}\left(x_{1}\right)\right| \leq \frac{1}{2}$. Note $x_{1} \notin B_{1}$, as $\left|V_{\epsilon}^{\prime}\right| \geq \frac{3}{4}$ on $B_{1}$. We repeat the same procedure for $V_{\epsilon}^{\prime}$ as for $V_{\epsilon}$, i.e., we choose a suitable ball centered at $x_{1}$, and of radius $\epsilon^{\beta}, \beta \in(\alpha, 2 \alpha)$ such that the corresponding inequality (3.9) is valid. We call this ball $D_{1}$, suppose $\operatorname{deg}\left(V_{\epsilon}^{\prime}, \partial D_{1}\right) \neq 0$, we stop. Otherwise we claim that we may replace $V_{\epsilon}^{\prime}$ inside $D_{1}$ by a minimizer with the same boundary value as $V_{\epsilon}^{\prime}$ on $\partial D_{1}$ and such that the energy will again drop by at least $C_{0}$. To see this, it suffices to observe that $V_{\epsilon}^{\prime}\left(x_{1}\right)=V_{\epsilon}\left(x_{1}\right)$ and, by (II), there is a ball of size $\cong \epsilon$ on which $\left|V_{\varepsilon}\left(x_{1}\right)\right|<\frac{3}{4}$. This ball will not interest any point $y$ for which $\left|V_{\varepsilon}^{\prime}(y)\right| \geq \frac{3}{4}$. In particular it will not interest the first ball $B_{1}$. Then we apply Lemma 2 , (3.21) to conclude the energy dropping fact. We repeat this process, and after a finite number of steps, we arrive at a ball $D_{N_{1}}$ with $\operatorname{deg}\left(V_{\epsilon}^{N_{1}}, \partial D_{N_{1}}\right) \neq 0$. Note the centers of all these balls $D_{j}$ are required to lie in side $B_{\delta}\left(a_{1}\right)$. We apply the same procedure to each $B_{\delta}\left(a_{j}\right), j=2, \ldots d$. Eventually, we find $d$-balls which we still call $B_{1}, \ldots B_{d}$ and a new map $\bar{V}_{\epsilon}$ as before with $\operatorname{deg}\left(\bar{V}_{\epsilon}, \partial B_{j}\right)=1, j=1,2, \ldots, d$, and $E_{\epsilon}\left(\bar{V}_{\epsilon}\right) \leq E_{\epsilon}\left(V_{\epsilon}\right)-c_{0}$, for a definite constant $c_{0}>0$.

We go back to the estimates (3.15), (3.16), and (3.17) to obtain the following: there is a point $b \in \Omega^{d}$, s.t. $\left|b_{j}-a_{j}\right| \leq \delta, j=1, \ldots, d$, and such that

$$
W_{g}(b) \leq W_{g}(a)-c_{0}+0(\rho) .
$$

By choosing $\rho \leq \delta^{2}<<1,(3.24)$ contradicts to the continuity of $W_{g}$ at $a \in \Omega^{d}$. This ends the proof of Theorem A.

\section{FINAL REMARKS}

Let a be a nondegenerate local minimum point for $W_{g}$, and let $w_{\epsilon}(x)$ be as chosen in the previous section. In particular $w_{\epsilon}$ satisfies (3.2). Then, as a consequence of the proof of Theorem $\mathrm{A}$, we see the solution $u_{\varepsilon}(x, t)$ of (1.1)-(1.3) with $u_{\epsilon}(x, 0)=w_{\epsilon}(x)$ has the following property: The set $\left\{(x, t) \in \Omega \times R^{+}:\left|u_{\epsilon}(x, t)\right| \leq \frac{1}{2}\right\}$ is contained in $\bigcup_{j=1}^{d} B_{\delta}\left(a_{j}\right) \times R^{+}$ whenever $\epsilon$ is sufficiently small (depending only on $\delta, g, \Omega$ and $\rho$ in the construction of $w_{\epsilon}$ ).

Vol. $12, \mathrm{n}^{\circ} 5-1995$. 
Now we consider the case that the set $K=\left\{b \in \Omega^{d}: W_{g}(b)=W_{g}(a)\right\}$ is not isolated points. From the property of $W_{g}, K$ is obvious a compact set in $\Omega^{d}$. We make the following assumption.

(4.1) The connected component $K_{a}$ of $K$, which contains the point a $\in \Omega^{d}$, is a smooth $k$-dimensional manifold, $1 \leq k \leq 2 d-1$. Moreover, for each point $b \in K_{a}, \nabla^{2} W_{g}(b)$ has $(n-k)$-positive eigenvalues.

From (4.1), we immediately see each $b \in K_{a}$ is a local minimum of $W_{g}$. Moreover, we have $\lambda_{0}=\inf \left\{\lambda_{b}, \lambda_{b} \neq 0\right.$ is an eigenvalue of $\left.\nabla^{2} W_{g}(b), b \in K_{a}\right\}$ is positive. Suppose $w_{\epsilon}(x)$ is chosen as before so that (3.2) is valid. Then we conclude from the proof of Theorem A that the solution $u_{\epsilon}(x, t)$ of (1.1)-(1.3) with the initial condition $u_{\epsilon}(x, 0)=w_{\epsilon}(x)$ has the property that the set

$$
\left\{(x, t) \in \Omega \times R^{+}:\left|u_{\epsilon}(x, t)\right| \leq \frac{1}{2}\right\}
$$

$\subseteq K_{a}^{\delta} \times R^{+}$whenever $\epsilon$ is sufficiently small. Here $K_{a}^{\delta}=\{x \in$ $\Omega$, dist $\left.\left(x, K_{a}\right) \leq \delta\right\}$. In particular, there is a point $a^{*}$ in $K_{a}$ and a sequence $\left\{u_{\epsilon}\right\}$ of steady state solutions of (1.1)-(1.2) such that

$$
\lim _{\epsilon \rightarrow 0} u_{\epsilon}=\prod_{j=1}^{d} \frac{x-a_{j}^{*}}{\left|x-a_{j}^{*}\right|} e^{i h(x)}
$$

for some harmonic function $h$ in $\Omega$.

Open Problem. - Can every point $b \in K_{a}$ have the property (4.2)? (Cf. also [BBH, problem 6]).

On the other hand, one can follow the proof of Theorem $A$ to show the following.

Corollary. - Let $K$ be a compact subset of $\Omega^{d}$, and $K_{\delta}=\{b \in$ $\left.\Omega^{d}: \operatorname{dist}(b, K)<\delta\right\}, \delta>0$. Suppose that $\inf \left\{W_{g}(b)=b \in \partial K_{\delta}\right\}>$ $\sup \left\{W_{g}(b): b \in K\right\}$. Then there is a family of steady state solutions $\left\{u_{\epsilon}\right\}, 0<\epsilon<\epsilon_{0}$ of (1.1)-(1.2) such that, for any sequence of $\epsilon_{n} \downarrow 0$, the sequence $u_{\epsilon_{n}}$ (up to subsequences) converges to $u^{*}(x)=$ $\prod_{j=1}^{d} \frac{x-a_{j}}{\left|x-a_{j}\right|} e^{i h(x)}, \Delta h=0$ in $\Omega, u^{*}=g$ on $\partial \Omega$, for some $a \in K_{\delta}$.

It is clear then $\nabla W_{g}(a)=0$. Next we consider the critical points of mountain-pass type. 
We assume that

$(H)$ there are two local minimum points $a$ and $b$ of $W_{g}$ such that

$$
\max \left\{W_{g}(a), W_{g}(b)\right\}<\inf _{\gamma \in \Gamma \cdot y \in \gamma([0,1])} \max _{g}(y)=c<\infty
$$

where

$$
\Gamma=\left\{\gamma \in c\left([0,1], \Omega^{d}\right): \gamma(0)=a, \gamma(1)=b\right\} .
$$

Then, it is well-known (cf. [R]) that there is a $\in \Omega^{d}, W_{g}(\mathbf{a})=c$ and $\nabla W_{g}(\mathbf{a})=0$. For $a, b \in \Omega^{d}$ as above, we can find two maps $u_{a}, u_{b}$ (as for (3.2)) such that

$$
\begin{aligned}
& E_{\epsilon}\left(u_{a}\right) \leq d I(\epsilon, \rho)+W_{g}(a)+\pi d \log \frac{1}{\rho}+0(\rho) . \\
& E_{\epsilon}\left(u_{b}\right) \leq d I(\epsilon, \rho)+W_{g}(b)+\pi d \log \frac{1}{\rho}+0(\rho) .
\end{aligned}
$$

Here $\epsilon<\epsilon(\rho), \rho<<\frac{1}{4} \min \left\{|b-a|, c_{0}\right\}, c_{0}=c-\max \left\{W_{g}(a), W_{g}(b)\right\}$.

We let $\boldsymbol{\Gamma}=\left\{\gamma \in c\left([0,1], H_{g}^{\prime}(\Omega)\right), \gamma(0)=u_{a}, \gamma(1)=u_{b}\right\}$, where

$$
H_{g}^{\prime}(\Omega)=\left\{u \in H^{\prime}(\Omega):\left.u\right|_{\partial \Omega}=g\right\} .
$$

We claim, for all sufficiently small $\epsilon>0$, that

$$
\inf _{\gamma \in \Gamma, u \in \gamma([0,1])} \max E_{\epsilon}(u)=\mathbf{c}>\max \left\{E_{\epsilon}\left(u_{a}\right), E_{\epsilon}\left(u_{b}\right)\right\} .
$$

Suppose for the moment that (4.5) is true, then, as the functional $E_{\epsilon}(\cdot)$ clearly satisfies the Palais-Smale condition (see $[\mathrm{R}])$ and $C^{1}$ on $H_{g}^{\prime}(\Omega)$, we conclude the following.

THEOREM C. - Suppose $W_{g}$ has a mountain-pass critical point in $\Omega^{d}$ so that $(H)$ is valid. Then there is a family $\left\{u_{\epsilon}\right\}, 0<\epsilon<\epsilon_{0}$ of critical points of (1.4) of mountain-pass type.

To show (4.3), we let $\gamma \in \Gamma$, and assume, without loss of generality, that $E_{\epsilon}\left(u_{b}\right) \leq E_{\epsilon}\left(u_{a}\right)$, and $W_{g}(b) \leq W_{g}(a)$. For any $v \in \gamma([0,1])$, we solve (1.1)-(1.3) with $u_{0}(x)=v(x)$. Denote $\mathbf{v}(x)=u(x, 1, v)$, where $u(x, t, v)$ is the solution of (1.1)-(1.3) with initial data $v$.

Then we find a new path connecting $u_{a}$ and $u_{b}$ as $u_{a}$ is connected to $\mathbf{u}_{a}$, $u_{b}$ is connected to $\mathbf{u}_{b}$ by the heat flow, and $\mathbf{u}_{a}$ is connected to $\mathbf{u}_{b}$ by the path $u(x, 1, v), v \in \gamma([0,1])$. We still denote this new path by $\gamma$. For any $s \in[0,1], \gamma(s)$ will satisfies, in the addition, that $\left|\nabla_{x} \gamma(s)\right| \leq \frac{C_{0}}{\epsilon}$, for some 
constant $C_{0}$. This follows from our choices of $u_{a}, u_{b}$ and the estimate (II). In fact, $C_{0}$ will only depend on $g$ and $\Omega$. It is also clear that $\max E_{\epsilon}(u)$ on this new path will be only smaller than $\max E_{\epsilon}(u)$ on the original path.

Let $K_{a}$ be the connected component of $\left\{\mathbf{b} \in \Omega^{d}: W_{g}(\mathbf{b})=W_{g}(a)\right\}$ containing $a$, and let $K^{\delta}$ be the set $\left\{\mathbf{b} \in \Omega^{d}: \operatorname{dist}\left(\mathbf{b}, K_{a}\right)<\delta\right\}, \delta>0$. By choosing $\delta>0$ so small that we have $K_{a}^{\delta} \subset \Omega^{d}$ and $\min \left\{W_{g}(\mathbf{b}), \mathbf{b} \in\right.$ $\left.\partial K_{a}^{\delta}\right\} \geq W_{g}(a)+C(\delta)$ for some $C(\delta)>0$.

Now we look at vortices of $\gamma(s), s \in[0,1]$. Let $s_{0}$ be the minimum of those $s \in[0,1]$ that there is a point $\mathbf{x}=\left(x_{1}, \ldots, x_{d}\right) \in \partial K_{a}^{\delta}$ with $x_{1}, \ldots x_{d}$ belonging to the set $\left\{y \in \Omega:\left|\gamma\left(s_{0}\right)(y)\right| \leq \frac{1}{2}\right\}$.

Then we claim $E_{\epsilon}\left(\gamma\left(s_{0}\right)\right)-E_{\epsilon}\left(u_{a}\right) \geq C(\delta)-0(\rho)>C(\delta)>0$ for all sufficiently small $\epsilon, 0<\epsilon<\epsilon_{0}<\epsilon(\rho)$, and $\rho$. Indeed, following the constructions in the proof of Theorem A, we have either

$E_{\epsilon}\left(\gamma\left(s_{0}\right)\right) \geq d I(\epsilon, \rho)+W_{g}(\mathbf{a})+\pi d \log \frac{1}{\rho}+0(\rho)+o(1)$, for some $\mathbf{a} \in \partial K_{a}^{\delta}$ or

$$
E_{\epsilon}\left(\gamma\left(s_{0}\right)\right) \geq d I(\epsilon, \rho)+W_{g}(\mathbf{a})+\pi d \log \frac{1}{\rho}+c_{0}+0(\rho)+o(1) .
$$

for some definite constant $c_{0}>0$ and some $\mathbf{a} \in K$. In either case, we have (4.5). This completes the proof.

\section{Complete the proof of Theorem B}

Finally we wish to verify $h(x, t)=h_{0}(x)$ which is needed in the proof of Theorem B. For a fixed time $t>0$, we look at the set $G(t)=\left\{x \in \Omega:\left|u_{\epsilon}(x, t)\right| \leq \frac{1}{2}\right\}$. Since $u_{\epsilon} \rightarrow u_{0}(x, t)$ weakly in $H_{\mathrm{loc}}^{\prime}\left(\bar{\Omega} /\left\{b_{1}, \ldots, b_{d}\right\} \times R_{+}\right)$and strongly in $L_{\mathrm{loc}}^{2}\left(\bar{\Omega} \mid\left\{b_{1}, \ldots, b_{d}\right\} \times R_{+}\right)$. We set that, for any $\delta>0, B_{\delta}\left(b_{j}\right) \cap G(t) \neq \phi$, and that $\operatorname{deg}\left(u_{\epsilon}(\cdot, t), \partial B_{\delta}\left(b_{i}\right)\right)=$ 1 for $j=1, \ldots, d$.

Let $x_{j} \in B_{\delta}\left(b_{j}\right) \cap G(t)$, and choose a ball $B_{j}$ of size $\epsilon^{\beta}, \alpha \leq \beta \leq 2 \alpha$, centered at $x_{j}$ so that the estimate similar to (3.9) is valid. We claim $\operatorname{deg}\left(u_{\epsilon}(\cdot, t), \partial B_{j}\right)=1$, for each $j=1, \ldots, d$. Otherwise, we simply follow the same argument as in the proof of Theorem A (for the case of local nondegenerate minimum) to obtain a new map $\bar{V}_{\epsilon}(x, t)$ such that

$$
E_{\epsilon}\left(\bar{V}_{\epsilon}\right) \leq E_{\epsilon}\left(u_{0}^{\epsilon}\right)-C_{0},
$$

for a definite positive constant $C_{0}$. At the same time,

$$
E_{\epsilon}\left(\bar{V}_{\epsilon}\right) \geq d I(\epsilon, \rho)+W_{g}(\bar{b})+\pi d \log \frac{1}{\rho}+0(\rho)+o(1),
$$


for some $\bar{b} \in \Omega^{d}$ with $\left|b_{j}-\bar{b}_{j}\right| \leq \delta$. We note that (4.3), (4.4), and (2.2) lead to a contradiction.

Thus we must have $\operatorname{deg}\left(u_{\epsilon}(\cdot, t), \partial B_{j}\right)=1$ for all $j=1, \ldots d$. Note that the estimate (2.14) will further imply that $x_{j} \rightarrow b_{j}$ as $\epsilon \rightarrow 0$, i.e., $\bar{b}=b$ in (4.4).

Similarly, we follow the previous arguments to obtain $\left|u_{\epsilon}(x, t)\right| \geq \frac{1}{2}$ on $\Omega_{\epsilon}=\Omega / \bigcup_{j=1}^{d} B_{j}$. (For otherwise (4.4) will be true as it was shown in Section 3.) We write $u_{\epsilon}(x, t)=\rho_{\epsilon}(x, t) \prod_{j=1}^{d} \frac{x-x_{j}}{\left|x-x_{j}\right|} e^{i h_{\epsilon}(x, t)}$ on $\Omega_{\epsilon}$, with $\left|\rho_{\epsilon}(x, t)\right| \geq \frac{1}{2}$. Note that $h_{\epsilon}(x, t)$ is uniquely determined up to $2 \pi(\bmod )$ on each $\partial B_{j}$. Since $\Omega_{\epsilon} \supseteq \Omega_{\delta}=\Omega / \cup_{j=1}^{d} B_{\delta}\left(b_{i}\right)$ for any $\delta>0$ whenever $\epsilon \leq \epsilon(\delta)$, we may rewrite $u_{\epsilon}(x, t)$ as $\prod_{j=1}^{d} \frac{x-b_{j}}{\left|x-b_{j}\right|} \tilde{\rho}_{\epsilon}(x, t) e^{i \tilde{h}_{\epsilon}}$ in $\Omega_{\delta} \times[0, T]$. We may also choose $\tilde{h}_{\epsilon}(x, t)$ so that it will be continuous in $t$ in $\Omega_{\delta} \times[0, T]$. It is easy to see, for any $\delta>0, \epsilon \leq \epsilon(\delta)$, that

$$
\int_{0}^{T} \int_{\Omega_{\delta}}\left|\frac{\partial}{\partial t} \tilde{h}_{\epsilon}\right|^{2} d x \leq 4\left(\int_{0}^{T} \int_{\Omega}\left|\frac{\partial}{\partial t} u_{\epsilon}\right|^{2} d x d t\right) \rightarrow 0
$$

from our choice of the initial data. Since $h_{\epsilon}(x, 0) \rightarrow h_{0}(x)$ in $H^{\prime}(\Omega)$ as $\epsilon \rightarrow 0$, we thus have $h_{\epsilon}(x, t) \rightarrow h_{0}(x)$ for a.e. $(x, t) \in \Omega \times[0, T]$.

\section{REFERENCES}

[BBH] F. BeTHUEL, H. BREZIS and F. HeLEIN, Ginzburg-Landau vertices, Birkhaüser, Boston, 1994.

[BBH2] F. Bethuel, H. Brezis and F. Helein, Asymptotics for the minimization of a Ginzburg-Landau functional, Cal. variations and P.D.E., Vol. 1\#2, 1993, pp. 123-148.

[BMR] H. BREZIS, F. MERLE and T. RIVIERE, Quantization effects, for $-\Delta u=u\left(1-(u)^{2}\right)$ in $\mathbf{R}^{2}$, preprint.

[CL] Y. M. ChEN and F. H. LIN, Evaluation of harmonic maps with the Dirichlet boundary condition, Comm. in Analysis and Geometry, Vol. 1\#3, 1993, pp. 327-346.

[CS] Y. M. CHEN and M. STRUwE, Existence and partial regularity for heat flow for harmonic maps, Math. Z, Vol. 201, 1989, pp. 83-103.

[HL] R. HARDT and F. H. LIN, Singularities for $p$-energy minimizing unit vector fields on planar domains, to appear in Cal. variation and P.D.E. 
[E] E. WEINAN, Dynamics of vortices in Ginzburg-Landau theories with applications to superconductivity, preprint.

[LSU] O. A. Ladyzenskaya, N. A. Solonnikov and N. N. URalseva, Linear and quasilinear equations of parabolic type, Translations of AMS, Mon. \#23 (196X).

[N] J. Neu, Vortex dynamics of complex scalar fields, Physics D., Vol. 43, 1990, pp. 384-406.

[PR] L. Pismen and J, RubinsteIn, Dynamics of defects, in nematics, mathematical and physical aspects, J. M. Coron et al. eds, Kluwer Pubs., 1991.

[R] P. RABINowITZ, Minimax methods in critical point theory with applications to differential equations, Conf. Board of the Math. Sci, by AMS \#65.

[RS] J. Rubinstein and P. Sternberg, On the Slow Motion of Vortices in the Ginzburg-Landau heat flow, preprint.

[S] L. Simon, Asymptotics for a class of non-linear evolution equations, with applications to geometric problems, Annals of Math., Vol. 118, 1983, pp. 527571.

[St] M. STRUwE, On the asymptotic behavior of minimizers of the Ginsburg-Landau model in 2 dimensions, J. Diff. Int. Eqs., Vol. 7, 1994.

[St2] M. STRUWE, On the evolution of harmonic maps of Riemannian surfaces, Comment. Math. Helv., Vol. 60, 1985, pp. 558-581.

(Manuscript received March 17, 1994;

revised May, 1994.) 